
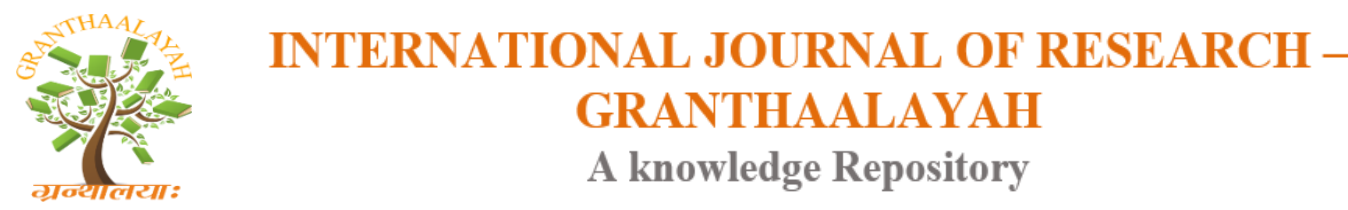

Science

\title{
INCARCERATED FEMORAL HERNIA PRESENTING AS CHRONIC NONSPECIFIC GROIN PAIN IN A YOUNG MALE - A VERY RARE CASE REPORT WITH LITERATURE REVIEW
}

\author{
Shariful Islam ${ }^{1,2}$, Vijaya Naidoo ${ }^{2}$, Malini Ramnarine ${ }^{1,2}$, Vinoo Bheem ${ }^{2}$, Vijay \\ Naraynsingh 1 \\ ${ }^{1}$ Department of Clinical Surgical science, University of the West Indies, St Augustine, Trinidad \\ and Tobago \\ ${ }^{2}$ Department of Surgery, San Fernando Teaching Hospital, San Fernando, Trinidad and Tobago
}

\begin{abstract}
Femoral hernia is uncommon of all groin hernias. It is commonly seen in elderly multiparous female patient. However, presentation of femoral hernia in a male patient is very rare. Only few cases of femoral hernia are reported in male patients in the English literature. Presentation of this hernia can be varied from asymptomatic to incarcerated groin lump. Diagnosis can often be very difficult on clinical examination alone especially in obese male patients. CT imaging should be used as an adjunct in making the right diagnosis when unsure. We present the case of a 42 year male COPD (Chronic Obstructive Pulmonary Disease) patient who visited several general practitioners for his chronic right groin pain with no relief. He was subsequently admitted to the General Hospital and a CT scan of the abdomen and pelvis was done which revealed a right femoral hernia. Emergency surgical repaired was performed. Literature search was performed and management of these hernias is highlighted.
\end{abstract}

Keywords: Femoral Hernia; Obese Patient; Male Femoral Hernia.

Cite This Article: Shariful Islam, Vijaya Naidoo, Malini Ramnarine, Vinoo Bheem, and Vijay Naraynsingh. (2018). "INCARCERATED FEMORAL HERNIA PRESENTING AS CHRONIC NONSPECIFIC GROIN PAIN IN A YOUNG MALE - A VERY RARE CASE REPORT WITH LITERATURE REVIEW.” International Journal of Research - Granthaalayah, 6(1), 448-454. https://doi.org/10.29121/granthaalayah.v6.i1.2018.1653.

\section{Introduction}

A femoral hernia is an uncommon condition which has been reported in less than $5 \%$ of all abdominal wall hernias, with a female to male predominance of $4: 1.60 \%$ of all femoral hernias are found on the right, $30 \%$ on the left, and $10 \%$ bilaterally [1]. There are only few reported cases of femoral hernias in the male patient in the English literature. 
Femoral hernias being uncommon are often times misdiagnosed as an inguinal hernia in the male population. Additionally, the examination and diagnosis based solely on clinical findings in the obese male can be difficult and misleading, thus CT imaging should be used as an adjunct in making the right diagnosis when unsure. It is important to exclude the diagnosis of a femoral hernia in the male patient as they are at higher risk of strangulation.

\section{The Case}

A 42 year old obese male COPD (chronic obstructive pulmonary diseases) patient presented to our accident and emergency department with sudden right groin pain after lifting a heavy object for 12 hours. The pain was associated with nausea but no vomiting. The patient had no urinary symptoms and had a normal bowel action on the morning of the admission. The COPD was well controlled by medicine. History revealed that the patient had this pain for several months for which he had visited several GP's and was treated with analgesic with little ease.

Physical examination revealed a middle age obese patient with no obvious cardiopulmonary distress. The mucous membranes were moist and pink. The vital examinations were normal. Abdominal examination revealed a large protuberant abdomen (Figure 1) with no visible mass or hernia in the right groin. However, mild tenderness was noted in that area on deep palpation. Rest of the abdominal and systemic examination were normal. Routine blood examinations were within normal limits except mild leucocytosis. Plain abdominal radiographs were normal. A CT abdomen and pelvis was done with intra venous contrast revealed a 5 x $5 \mathrm{~cm}$ right femoral hernia with a $3 \mathrm{~cm}$ diameter neck containing omentum only (Figure 1).

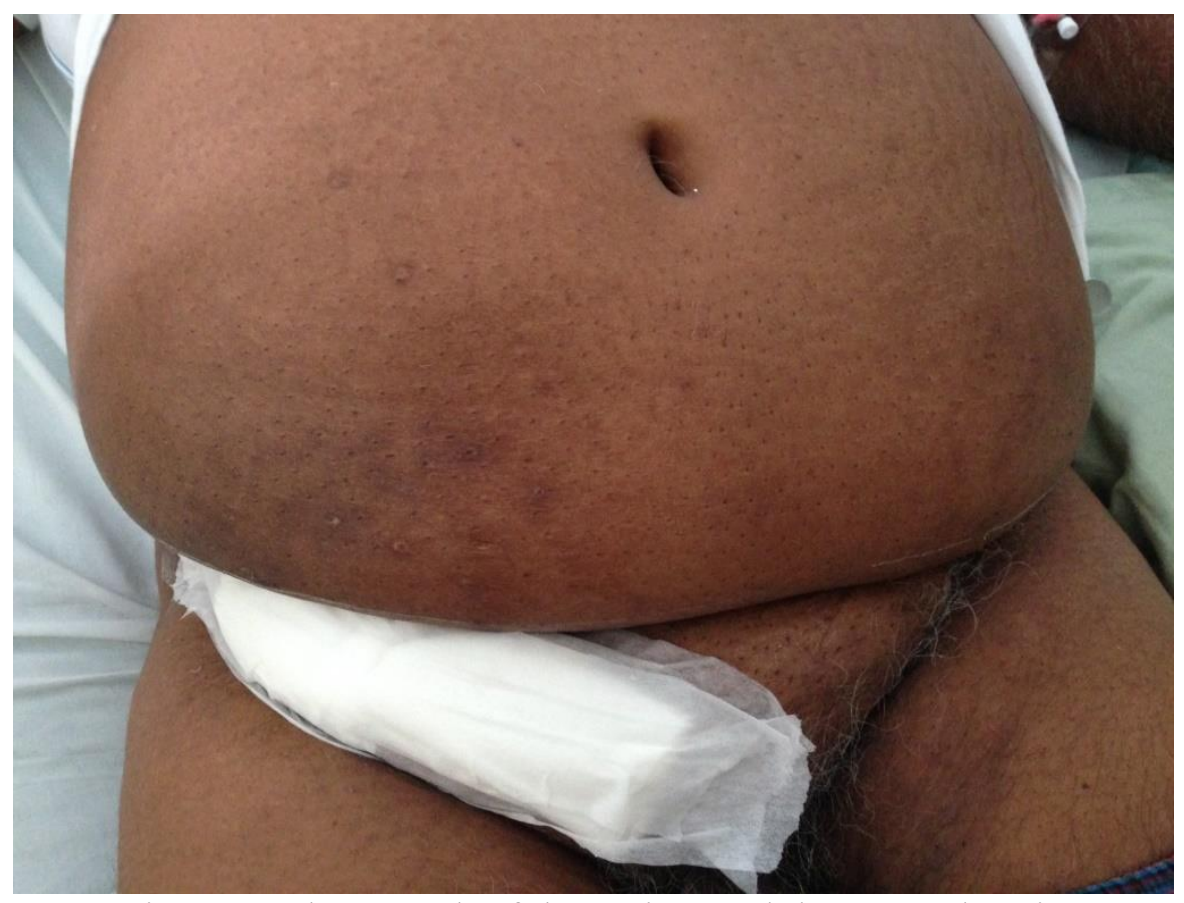

Figure 1: Photograph of the patient's abdomen and groin 


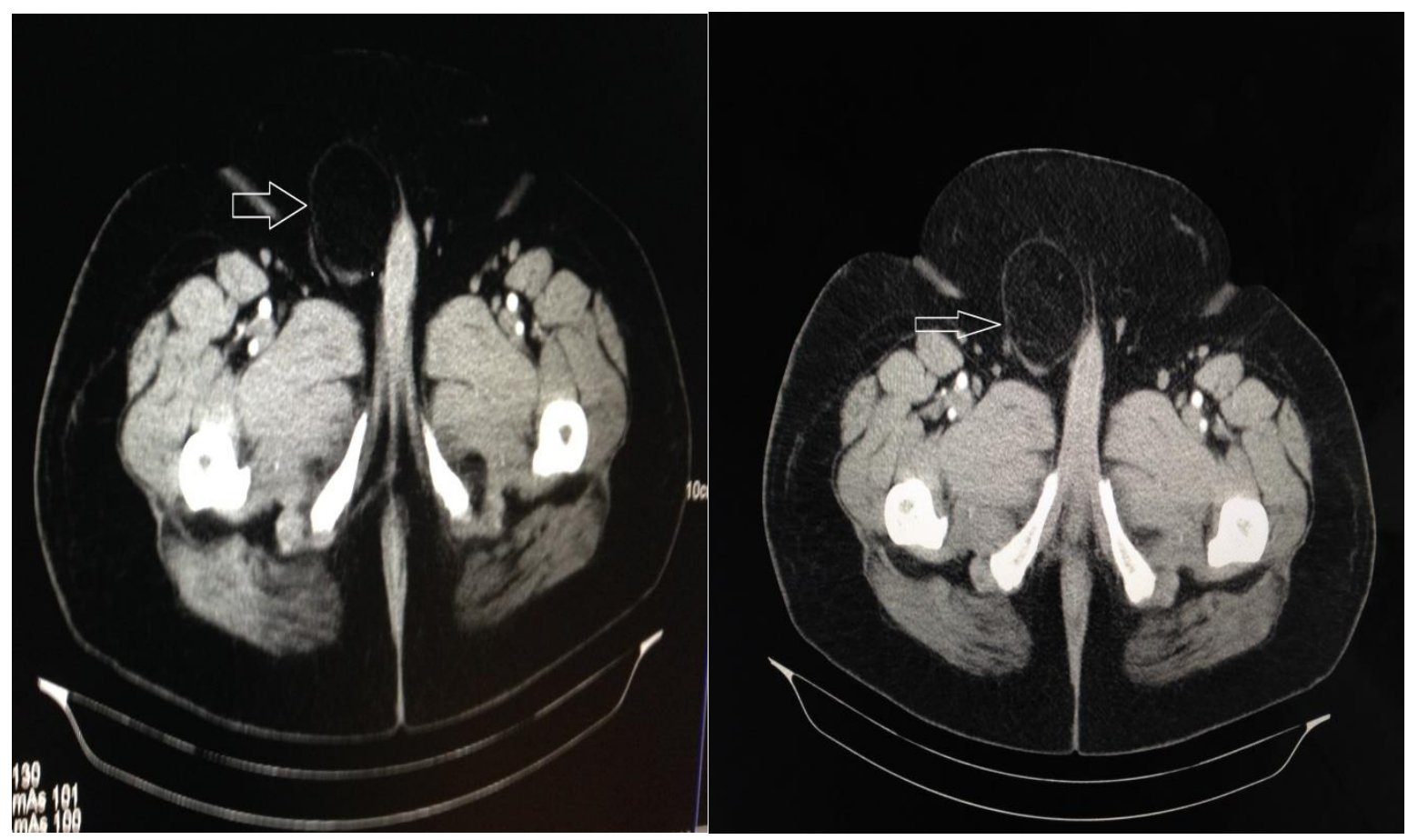

Figure 2: CT scan of abdomen and pelvis with intravenous contrast demonstrating the thick abdominal wall with a $5 \times 5 \mathrm{~cm}$ right femoral hernia

The patient was resuscitated and optimized prior to emergency inguinal exploration under spinal anaesthesia. A trans-inguinal approach was used. Intraoperative findings revealed a $5 \mathrm{~cm} \times 5 \mathrm{~cm}$ right femoral hernia protruding posterior to the inguinal ligament (Figure 3). The sac was opened and contained oedematous omentum (Figure 4). Partial omentectomy was done and the posterior wall of the inguinal canal was repaired. The femoral canal was closed by two interrupted sutures using 1'0 prolene. Mesh hernioplasty was then performed using 2'0 prolene.

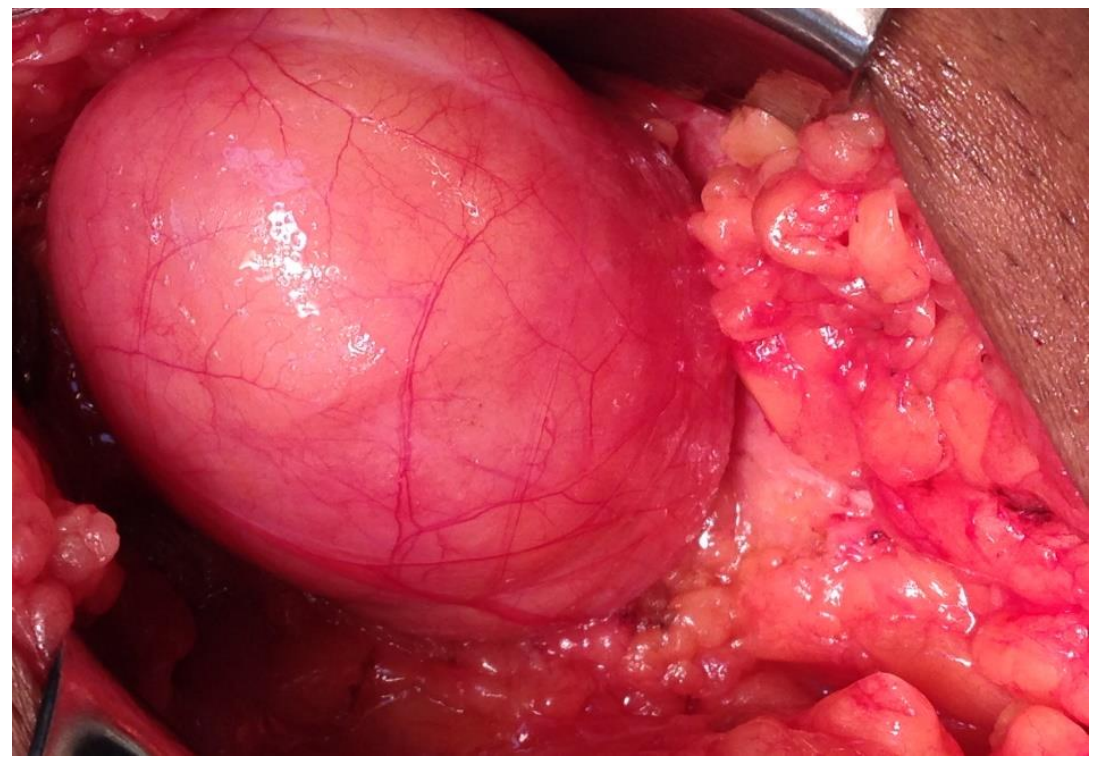

Figure 3: Intraop photograph of the large femoral hernia sac 


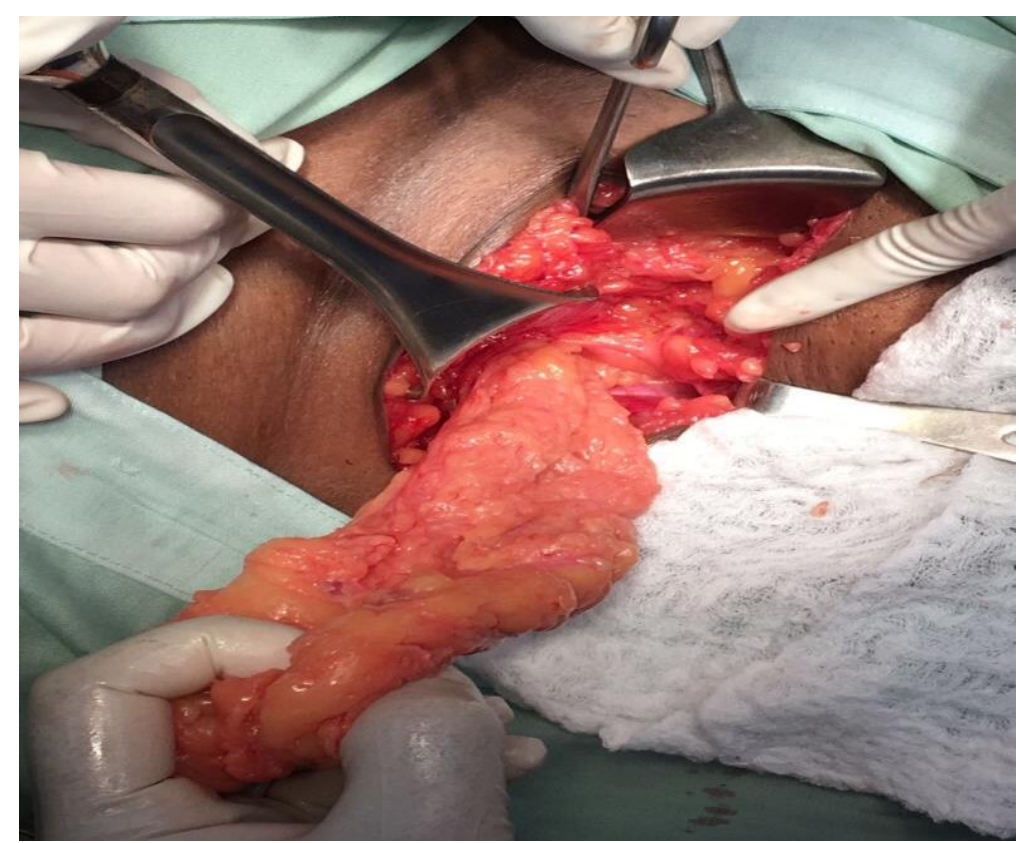

Figure 4: Intraop photograph showing omentum as the content of the sac

The patient had an unremarkable post op recovery and was discharged on day 3 postsurgical repair. Patient has been followed up in the surgical outpatient clinic and at 2 years follow up the patient was doing fine with nil complaints or sings of recurrence.

\section{Discussion}

Femoral hernias are a relatively uncommon, accounting for less than $5 \%$ of all hernias, with a female predisposition, likely due to the wider pelvis or wider femoral canal. The male to female ratio is 1: 4 and increases with age. [1] The peak age distribution is 50 years and older [2] however there have been only 2 reported cases of femoral hernias presenting in the younger male ages 24 and 40. These cases were found to have bilateral femoral hernias [3, 4]. Literature search also revealed 3 other cases of unilateral male femoral hernias however all of them are over the age 57 and over.[5-7] Two of these cases were presented as De Garengeot hernias. [6, 7] Our patient being 42 year is the $3^{\text {rd }}$ youngest case of male femoral hernia but the first youngest case of unilateral femoral hernia.

Many theories have been postulated with regards to the aetiology of the femoral hernia however the most fundamental of them all is based on the premise of an enlarged femoral ring. An anatomical study was done by Tasche which revealed the variation of the narrow posterior inguinal wall attachment on to the pectineal ligament, resulting in an enlarged femoral ring and resulting femoral herniation. [8]

Other risk factors are: Obesity, chronic cough or chronic constipation, enlarged prostate, improper heavy lifting; elderly, low weight women have an increased risk than individuals of any other age group. Rarely, a congenital anatomical weakening can increase the risk these however present in the Paediatric population. 
Diagnosing femoral hernias preoperatively is challenging due to the resemblance to the inguinal hernia. Diagnosing the nature of a lump in the groin is often difficult and a delay in diagnosis may occur, especially because an obstructed or strangulated femoral hernia may not always typically present with abdominal or inguinal pain. There are 2 published cases of bilateral femoral hernias.[3, 4] The patients presented with a right groin swelling, one of which was diagnosed clinically having a bilateral hernia and the other, unilateral. They both proceeded to have emergency surgery without imaging, based on clinical findings. 3 months post operatively the unilateral repair was found to have a left femoral hernia for which he then proceeded to have an elective repair. Despite the diagnosis being primarily a clinical one, the use of CT imaging as an adjunct is undeniably useful. On axial CT images, the neck of the femoral hernia sac can be seen as a narrow protrusion through the femoral ring just medial to the common femoral vein, which often appears compressed by the sac. Femoral hernias also have a characteristic funnelshaped neck and the inguinal canal which is medial to the sac, may appear oedematous. These hallmark features makes CT imaging an excellent adjunct in diagnosing the femoral hernia and also ensures that a bilateral femoral hernia is not missed. A retrospective review was done to investigate the CT findings of femoral hernias, focusing on their differentiation from inguinal hernias. On review of 46 femoral hernias, 45 cases were detected on CT. In all 45, hernia sacs were localized and 42 lesions showed venous compression. In the review of 215 groin hernias, all 11 femoral hernias had localized sacs with venous compression on CT. Of the 204 inguinal hernias, 98 lesions were detected on CT, 65 had extended sacs, and only 10 showed venous compression. Localized sacs with venous compression were seen much more often in the femoral hernias $(11 / 11,100 \%)$ than in the inguinal hernias $(1 / 92,1.1 \%)$. [9] It was thus concluded that CT imaging is accurate in differentiating inguinal and femoral hernias. Our patient was presented with chronic nonspecific groin pain. He had no clinically detectable lump in the groin other than mild tenderness. The CT scan of the abdomen and pelvis helped us in the diagnosis of the femoral hernia.

The optimal management of femoral hernia is surgical. There is no debate or controversy on that. It is performed urgently in patients who present with acute incarceration or strangulation. For patients with no complications, the optimal timing of repair (watchful waiting versus early elective repair) and the optimal surgical technique are controversial. In patients with a newly diagnosed femoral hernia, elective surgical repair is recommended, rather than watchful waiting, regardless of the patient's sex and symptoms, as they are associated with a high risk of complications. Femoral hernias, when compared to inguinal hernias are associated with a higher risk of developing complications. In one study, Gallegos NC et al reported the rates of strangulation were 22 and 45 percent at 3 and 21 months post initial diagnosis, respectively, for femoral hernias, compared with 2.8 and 4.5 percent for inguinal hernias.[10] Thus, early elective repair is advised for patients with a newly diagnosed femoral hernia to avoid complications that may arise from emergency surgery. Urgent surgery for complicated hernias is more likely to involve bowel resection, which is associated with a higher mortality rate. In one study, bowel resection was required in 23 percent of urgent, compared with 0.6 percent of elective femoral hernia repairs, and urgent femoral hernia repairs were associated with a 10 -fold increase in mortality. [11] This lends support to early elective surgical management once diagnosed.

The surgical approach depends on both hernia and patient characteristics. Both open and laparoscopic techniques have been described however much controversy still exits regarding 
which approach should be taken. Three open approaches are described to repair a femoral hernia; the Lockwood (low approach), McEvedy (high approach), or Lotheissen's (Trans inguinal approach). There are two laparoscopic approaches, which include the totally extra peritoneal approach (TEP) or the trans-abdominal preperitonoeal approach (TAPP).

Laparoscopic repair has been associated with lower recurrence rates and postoperative pain compared to the open techniques. A prospective cohort study was conducted on femoral hernia repairs registered in the Danish Hernia Database from January 1998 to February 2012. A total of 3970 primary femoral hernia repairs were analysed. In a multivariate analysis, laparoscopic repair was found to result in reduced risk of reoperation compared to the open repair. [12] A Cochrane review published in 2008, showed that hernia recurrence post laparoscopic mesh repair was less common compared to open, non-mesh repair, with the main indicator of recurrence related to the use of a mesh rather than the approach itself.[13] Despite its advantages, laparoscopy requires more time, specialised skill and is more expensive.

\section{Conclusion}

Femoral hernias in the male still remain a rare occurrence. In the male patient presenting with pain or a swelling to the groin, one must ensure to examine thoroughly to exclude the diagnosis of a femoral hernia as despite its rarity can and still lead to grave complications if not diagnosed and surgically repaired in a timely fashion. The diagnosis relies heavily on the expertise of the clinical examination however if unsure, one must not hesitate to make use of CT imaging to aid in the correct diagnosis.

\section{Learning Points}

1) Femoral hernias are very rare in males and are usually occur in the older male with predisposing risk factors.

2) Male patient less than 50 years of age who presents with a unilateral femoral hernia; a thorough examination should be done to look for contralateral hernia.

3) CT imaging is of great value to diagnose the femoral hernia (uni or bi lateral) as well as for other differentials particularly in obese patients.

4) Symptomatic femoral hernia should be repaired immediately as undue delay will increase the risk of bowel resection.

5) Asymptomatic femoral hernias on the other hand should be at the earliest elective date once diagnosed. There is no role for watchful waiting.

\section{Conflicts of Interest}

The authors declare no conflicts of interest.

\section{Author Contribution}

All authors have contributed significantly in designing and organizing to write manuscript, collecting data as well help in critical analyzing the manuscript. All authors have approved the final version of this manuscript. 


\section{Funding}

No fund was received to publish this article.

\section{Consent}

Patient consent was obtained.

\section{Guarantor}

The corresponding author will accept the full responsibility for the work.

\section{References}

[1] Mcintosh A, Hutchinson A, Roberts A, and Withers H. (2000). 'EVIDENCE BASED MANAGEMENT OF GROIN HERNIA IN PRIMARY CARE-A SYSTEMATIC REVIEW'. Fam Pract; 17:442.

[2] Sucandy I, and Kolff JW. (2012). 'INCARCERATED FEMORAL HERNIA IN MEN: INCIDENCE, DIAGNOSIS AND SURGICAL MANAGEMENT'. North Am J Med Sci. 4(11):617-8. Doi: 10.4103/1947-2714.103343.

[3] Kouchupapy R, Ranganathan G, Dias S, and Shanahan D. (2013). 'AETIOLOGY OF FEMORAL HERNIAS REVISITED: BILATERAL FEMORAL HERNIA IN A YOUNG MALE (TWO CASES)'. Annals of the Royal College of Surgeons of England. 95(1):e14-e16. Doi: 10.1308/003588413X13511609955733.

4. Rahaman QM. (2005). 'BILATERAL FEMORAL HERNIA IN A MALE-A CASE REPORT'. Journal of Indian Med Association.

[4] Mahajan A, and Luther A. (2014). 'INCARCERATED FEMORAL HERNIA IN MALE: A RARE CASE REPORT'. Int Surg J. 1(1): 25-26. Doi:10.5455/2349-2902.isj20140508

[5] Túlio FL, Carlos AA. Chagas, Lucas ASP, Rafael C, and Márcio AB. (2015). 'DE GARENGEOT'S HERNIA IN AN 82 YEAR OLD MAN: A CASE REPORT AND CLINICAL SIGNIFICANCE'. International Journal of Surgery Case Reports. Vol.17:85-88, doi:10.1016/j.ijscr.2015.10.031

[6] Abdul W, Thomas C, and Swarnkar K. (2015). 'GARENGEOT'S HERNIA: A RARE PRESENTATION OF AN ISCHEMIC APPENDIX WITHIN A STRANGULATED FEMORAL HERNIA IN AN ELDERLY MALE'. International Journal of Scientific research Vol 4, No 9

[7] Mcvay CB, and Savage LE. (1961). 'ETIOLOGY OF FEMORAL HERNIA'. Annals of Surgery. 154(Suppl 6):25-32.

[8] Suzuki S, Furui S, Okinaga K, Sakamoto T, Murata J, Furukawa A, and Ohnaka Y. (2007). 'DIFFERENTIATION OF FEMORAL VERSUS INGUINAL HERNIA: CT FINDINGS'. American Journal of Roentgenology 189:2, W78-W83

[9] Gallegos NC, Dawson J, Jarvis M, and Hobsley M. (1991). 'RISK OF STRANGULATION IN GROIN HERNIA'. Br J Surgery. 78:1171.

[10] Dahlstrand U, Wollert S, and Nordin P, et al. (2009). 'EMERGENCY FEMORAL HERNIA REPAIR: A STUDY BASED ON A NATIONAL REGISTER'. Ann Surg; 249:672.

[11] Andresen K, Bisgaard T, Kehlet H, Wara P, and Rosenberg J. (2014). 'REOPERATION RATES FOR LAPAROSCOPIC VS OPEN REPAIR OF FEMORAL HERNIA IN DENMARK: A NATIONWIDE ANALYSIS'. JAMA Surg. 149(8):853-7. Doi: 10.1001/jamasurg.2014.177

[12] Mccormack K, Scott N, Go PM, Ross SJ, and Grant A.(2003). 'COLLABORATION OF THE EU HERNIA TRIALISTS. LAPAROSCOPIC TECHNIQUES VS OPEN TECHNIQUES FOR INGUINAL HERNIA REPAIR. COCHRANE DATABASE OF SYSTEMIC REVIEWS'. Issue 1. [DOI: 10.1002/14651858.CD001785]

*Corresponding author.

E-mail address: sss1201198@ yahoo.com/shar_islam7@ hotmail.com 